\title{
CONTROL CENTRAL Y SINTOMATOLOGÍA PSICÓTICA
}

\author{
Pedro C. Martínez Suárez, Serafín Lemos Giráldez, Mercedes Paíno \\ Piñeiro, Ana María López Rodrigo, Mercedes Inda Caro y Juan A. Gil-López \\ Universidad de Oviedo
}

\begin{abstract}
RESUMEN
En ocasiones, nuestro organismo interpreta determinadas formas de lenguaje interno como voces procedentes del exterior. El presente trabajo tiene el objetivo de averiguar si aquellos pacientes que presentan alucinaciones auditivas difieren de otros grupos clínicos en las funciones de discriminación/atribución de la fuente estimular . Los sujetos fueron clasificados según su sintomatología (Brief Psychiatric Rating Scale). Como variable criterio se contabilizaron los errores en una prueba de control central construida a tal efecto. De este estudio, únicamente se deducen diferencias en control central bajo aquellas condiciones de generación verbal en que el sujeto ve restringida su producción. Situación ésta en la que los sujetos con alucinaciones auditivas tienden a atribuir falsamente a agentes externos sus propias producciones. Posteriores investigaciones deberían explorar con más precisión las funciones verbales y motoras (voluntariedad/involuntariedad de la acción) que definen los procesos de control.
\end{abstract}

Palabras clave: control central, alucinaciones auditivas, discriminación/atribución de la fuente estimular.

\begin{abstract}
Sometimes, our organism interprets specific types of internal speech as voices coming from the outside. The aim of this study was to discover if patients with auditory hallucinations differ from other clinical groups in the discrimination/ attribution about the origin of the stimuli. Subjects were classified according to their symptomatology (Brief Psychiatric Rating Scale).The errors in a central monitoring task were considered as criterion variable. Only differences were found in monitoring under those conditions of verbal generation where subject's production is restricted. In that situation, psychotic patients with auditory hallucinations attribute erroneusly to an external agency their own productions. Further research should explore verbal and control motor functions (voluntary and non voluntary actions) that define processes of monitoring more accurately.
\end{abstract}

Key words: monitoring, auditory hallucinations, discrimination/attribution, origin of the stimuli.

Correspondencia: D. Serafin Lemos. Facultad de Psicologia. Universidad de Oviedo. Tel. (98) 5103254. C/ Aniceto Sela, $s / n^{\circ} .33005$ Oviedo 


\section{INTRODUCCIÓN}

El término psicótico ha bordeado históricamente la polisemia. Incluso desde el punto de vista técnico ha sufrido ciertas variaciones. Sin embargo, los cambios que pueden observarse en el DSM-IV (Asociación Americana de Psiquiatria, 1994) o el ICD-10 (Organización Mundial de la Salud, 1992), con respecto a sus predecesores DSM-III e ICD-9, no han sido de tipo semántico sino lógico. Así, mientras que en las clasificaciones anteriores se ha venido equiparando el término psicosis funcional a desorden mental, caracterizándolo por alteraciones del juicio y distorsión de lo real, actualmente se operativiza tal concepto otorgando un papel protagonista a ciertas experiencias (llamadas positivas) como soñ: alucinaciones y/o delirios. Por alucinaciones se entiende toda experiencia sensorial que posee la viveza de realidad de una verdadera percepción pero que ocurre en ausencia de estimulación real del órgano sensorial relevante (Asociación Americana de Psiquiatría, 1994, p. 766). El delirio se define como una falsa creencia basada en inferencias incorrectas acerca de la realidad externa y que son mantenidas con firmeza a pesar de ser opuesta a las creencias de la población general y de tener contundente evidencia en contra (Asociación Americana de Psiquiatria, 1994, p. 765). La clave de este cambio ha estado en el paso de una lógica descendente o si se quiere deductiva, a una lógica ascendente 0 inductiva.

Tal vez, para añadir nitidez a la definición, el DSM-IV rebautiza circularmente el término psicosis acudiendo a nuevas formas para viejos contenidos, esto es, considera la aparición de experiencias alucinatorias y delirantes como elementos de un conjunto conceptual que implica disolución del "yo" y grave deterioro en el enjuiciamiento de la realidad.

Contemplando las últimas hipótesis explicativas del fenómeno psicótico, parece propio sospechar una fuerte complicidad entre los procesos de enjuiciamiento de la realidad o prueba de realidad y fenómenos como las alucinaciones, propios de la sintomatología psicótica.

A finales del siglo pasado resultaron especialmente productivas las teorias que partiendo de las funciones perceptivas, mnemónicas o imaginativas pretendian explicar el fenómeno de las alucinaciones (Tuke, 1889). Pero es con William James y Alfred Binet con quien se plantea la polémica sobre el origen corticalcentral o periférico de las alucinaciones. Asi, Binet mantiene la posición de que las corrientes de los nervios periféricos serían las únicas capaces de encender el proceso máximo o supraideacional que hará que el objeto percibido tenga el carácter de externalidad. Mientras que por otro lado, James (1890) argumenta que en cierto tipo de alucinaciones $y$ pseudoalucinaciones se produce una "inhibición central" que impide alcanzar el grado de realidad adecuado. Por otra parte, es Freud (1895) quien introduce el concepto "signo de realidad" el cual lleva la información que distingue entre la percepción y la representación de un objeto.

Hasta ahora, hemos visto como las experiencias psicóticas de tipo positivo, sobre todo las alucinaciones, son interpretadas como alteraciones en un proceso que implica: a) discriminación entre lo percibido y lo pensado y b) discriminación entre lo externo y lo interno. Este proceso (que en la presente investigación se llama "control central") recibe varias acepciones, tanto en la literatura clásica ("inhibición central", "signo de realidad"), como en los sistemas de clasificación modernos ("prueba de realidad"). Pero además, en nuestros dias, el tema de las alucinaciones y los delirios es abordado desde la horizontalidad teórica de innumerables trabajos neuropsicológicos. Nos centraremos en aquellos que afrontan la sintomatología positiva desde el punto de vista del control central (Gray, Feldon, Rawlins, Hemsley y Smith, 1991; Frith, 1992).

La teoria de Hoffman (1986), tiene que ver con la planificación del discurso y las intenciones comunicativas del sujeto. Esta teoria se puede resumir en cuatro puntos: a) las propiedades sensoriales de las alucinaciones verbales no difieren de aquellas que conlleva la mera imaginación (la gran mayoría de los estudios que aporta en este sentido han sido realizados con sujetos esquizofrénicos); b) las alucinaciones auditivas podrian ser imágenes que deben seguir una compleja serie de pasos con diferentes grados de consciencia, pudiendo existir alteraciones en uno o varios de estos pasos (parte central de la teoria); c) diversos estudios en inteligencia artificial revelan la relación entre alucinaciones verbales y desorganización verbal, de modo que la falta de se- 
cuenciación en la planificación lingüistica puede coincidir con la aparición de errores no sometidos a control voluntario muy similares a las imágenes propias de las alucinaciones; d) estas imágenes no concordantes con la planificación voluntaria $y$, por tanto, de orden inconsciente, producen las sensaciones de extrañeza y alienación propias de las alucinaciones.

De gran interés resulta el modelo propuesto por Slade y Bentall (1988), conocido como modelo de los cinco factores, teoria ad hoc de la original de Slade (1976) compuesta por cuatro factores. Esta teoria considera las alucinaciones como resultado de fallos en la habilidad de discriminación de la realidad, llevando al sujeto a atribuir falsamente pensamientos o verbalizaciones internas a una fuente externa. Con esta teoria se pretende dar cuenta de: a) las observaciones que detectan actividad subvocálica durante las alucinaciones; b) el efecto producido por tareas verbales concurrentes inhibiendo el discurso interno y provocando alucinaciones auditivas; c) la existencia de este tipo de experiencias en sujetos normales; y d) el carácter dimensional del fenómeno alucinatorio. Los cinco factores a los que se refieren los autores son:

1.- Estrés-activación: circunstancias ambientales, especialmente referidas a un ambiente familiar hostil que pueden desencadenar el proceso alucinatorio. Esto conlleva un estilo de selección de la información y de tratamiento del estímulo tendente a fijar las características semánticas y focalizar la atención únicamente en las caracteristicas físicas.

2.- Factores de predisposición, relativos a alteraciones de tipo cognitivo subyacentes. Deben ser investigados aquellos déficits que tienen que ver con la discriminación de la realidad: juicio sobre el origen de las propias percepciones, discriminación de la información relevante, localización espacial de sonidos y en especial la habilidad para utilizar pistas en la elaboración de estrategias cognitivas.

3.- Estimulación ambiental: situaciones de deprivación y ruido desorganizado. El juicio acerca de si un hecho es real o imaginario es probable que se complique cuando las propiedades sensoriales de ambos ambientes (interno-externo) son similares. Estas situaciones de ambigüedad facilitan la aparición de alucinaciones auditivas.
4.- Refuerzo: en algunos casos en los que se produce un decremento del arousal tras la experiencia alucinatoria, este efecto parece aliviar el proceso mientras que en casos en que hay un incremento en el arousal parecen pronunciarse los episodios. Al respecto, los autores son muy prudentes al indicar que es necesario ampliar el estudio del reforzamiento para concluir con claridad que el juicio de realidad conlleva a determinadas consecuencias conductuales.

5.- Expectativa: las creencias del sujeto y el grado en que las alucinaciones afectan a esta esfera (refleja la relevancia de la conexión, al menos teórica, entre alucinaciones y delirios).

No obstante, el proceso de discriminación que permite diferenciar entre lo percibido y lo pensado, lo externo y lo interno ha sido incorporado por algunos autores a un supuesto mecanismo que dependería de instancias corticales superiores. Según este proceder se han formulado varios modelos.

El modelo de Hemsley (Hemsley, 1987 y 1993; Peters, Pickering y Hemsley, 1994) parte del funcionamiento perceptivo normal. Los modelos de cognición normal aceptan que la percepción depende de la interacción entre los estímulos presentes y los recuerdos almacenados de regularidades (experiencias) sensoriales previas. La información contextual actual en conjunción con las regularidades previas da lugar a las expectativas o sesgos de respuesta.

La condición esquizofrénica se caracteriza por un debilitamiento de la influencia ejercida por las regularidades sensoriales pasadas en la percepción actual. Las alucinaciones estarian relacionadas con una disfunción cognitiva que bajo condiciones normales derivaría en mensajes que alcanzan elevados niveles de consciencia y por tanto son incapaces de inhibir material procedente de la memoria a largo plazo permitiendo su intrusión y siendo percibido este material como procedente de una fuente externa. Durante las alucinaciones auditivas el fallo se produciria en la capacidad inhibitoria del "input" sensorial actual sobre las regularidades perceptivas almacenadas, podria tratarse de un fallo del sistema perceptivo o un fallo de los sistemas de almacenamiento a corto plazo que manejan la información sensorial (Hemsley, 1987). 
Otro modelo que conecta igualmente la sintomatologia psicótica positiva (especialmente alucinaciones auditivas) con alteraciones en un sistema de control central es el modelo de Frith (Frith, 1987, 1992; Frith y Done, 1988, 1989; Mlakar, Jensterle y Frith, 1994), en el que se postula que una acción puede ser elicitada a través de dos rutas: la primera, basada en la planificación y en la memoria a largo plazo (el propio autor discute hoy este punto) mediante la cual se forman las acciones voluntarias; la segunda, basada en la estimulación externa y también consultativa de la memoria a largo plazo, conduce a la formación de una respuesta provocada por un estímulo externo. La existencia de un circuito de retroalimentación informa a un supuesto "monitor" (controlador), mecanismo supervisor central, sobre la voluntariedad o elicitación externa de la acción, así como de su realización efectiva. La corriente de re-aferencia encargada de transmitir esta información sería similar a la descarga corolaria ocular. Frith (1987) supone la existencia de esta descarga aplicada también a los pensamientos. $\mathrm{Si}$, por cualquier causa, este flujo informativo es alterado y consecuentemente no son adecuadamente controladas las acciones voluntarias del sujeto esto dará lugar a estas experiencias: a) que la estimulación proceda de una fuente externa (alucinación); b) que las conductas sean percibidas como iniciadas por agentes externos (experiencias de pasividad: delirios de control, inserción de pensamiento, etc.); y c) que las acciones sean juzgadas como consecuencias de estímulos inconexos (delirios de referencia).

El denominador común de las teorias mencionadas radica en considerar las alucinaciones auditivas como imágenes, verbalizaciones o pensamientos cuyo origen está en el propio sujeto pero que el organismo interpreta como ajenos a él, lo cual se produce al fallar un mecanismo de control central que pudiera tener funciones inhibidoras (modelo de Hemsley) o informadoras (modelo de Frith).

Se hace necesario acudir a estudios fuera del ámbito clínico (Johnson, Taylor y Raye, 1977; Johnson y Raye, 1979; Johnson, Raye, Hasher y Chromiak 1979; Johnson y Raye, 1981; Raye y Johnson, 1980; Johnson, Raye y Durso, 1980; Johnson, Hashtroudi y Lindsay, 1993; Raye, Johnson y Taylor, 1980) para aclarar el/los constructo/os teórico/os que pretende/en dar cuenta del proceso alucinatorio.

Según Johnson y Raye (1981) habria que distinguir entre: a) "control de la realidad": proceso mediante el cual atribuímos el origen de un recuerdo a una fuente externa o interna; y b) "prueba o juicio de realidad": distinción en tiempo presente entre una percepción y un acto de imaginación (su disfunción sería según la Asociación Americana de Psiquiatría [1994, p. 770], lo que caracterizaría el término psicótico).

Normalmente es difícil distinguir en la literatura, a no ser por las tareas utilizadas, si un investigador se refiere a uno $u$ otro concepto. Johnson y Raye (1981) diseñan una serie de pruebas para medir el constructo "control de la realidad" consistentes en alternar palabras generadas por el sujeto y palabras generadas por el experimentador; transcurrido un periodo de tiempo le presentan al sujeto una lista de reconocimiento con las palabras generadas para que haga una atribución según crea que la palabra ha sido generada por el experimentador o por él mismo.

Posteriormente, analizan el número y tipo de errores que el sujeto comete. Algo muy similar ha sido puesto en práctica en población clínica por Harvey (1985), este autor encuentra diferencias significativas entre un grupo de esquizofrénicos y maníaco-depresivos con sintomatología positiva y un grupo control.

Lo que en esta investigación se llama control central sería equivalente a lo que Johnson y Raye (1981) llaman control de la realidad, sin embargo, pretende ser validado en el marco teórico del modelo de Frith (1987) de ahi que se haya sustituido el término "de la realidad" por "central".

El objetivo del presente estudio ha consistido en detectar posibles diferencias en el primero de los aspectos del control central, entre cuatro grupos de población clínica, analizando en particular las diferencias existentes entre aquellos pacientes que presentan alucinaciones auditivas $y$ aquellos que no las presentan. A tenor del modelo sugerido por Frith (1987), los pacientes que presentan alucinaciones auditivas cabe esperar que cometan más errores atribucionales en la fuente del estímulo, comparativamente con los no alucinadores, atribuyendo falsamente a una fuente externa los estímulos autogenerados. 


\section{MÉTODO}

\section{Sujetos}

La muestra se compone de 40 sujetos ( $67,5 \%$ mujeres y un $32,5 \%$ hombres) con edades comprendidas entre los 18 y 72 años, la media es de 42,1 años y la desviación típica 11,7 años. Un $42,5 \%$ de los pacientes autoinformaron estudios primarios, un $40 \%$ bachillerato o equivalente, un $10 \%$ sin estudios y un $7,5 \%$ estudios universitarios. Los sujetos fueron evaluados en una consulta ambulatoria de neuropsiquiatria perteneciente a la red sanitaria pública. Se constituyeron cuatro grupos de 10 unidades: el primer grupo formado por pacientes psicóticos que presentaban alucinaciones auditivas en el momento de la realización de la prueba (5 diagnosticados de esquizofrenia paranoide y 5 de esquizofrenia indiferenciada), el segundo formado por pacientes igualmente psicóticos pero que no presentaban alucinaciones auditivas en el momento de realizar la prueba (8 diagnosticados de trastorno delirante y 2 de esquizofrenia residual), un tercer grupo de pacientes con trastornos afectivos ( 9 con trastornos depresivos y 1 con trastorno bipolar) y un cuarto grupo compuesto por pacientes con diversos trastornos de ansiedad.

La orientación terapeútica fue totalmente médica. Los pacientes que comprendieron los grupos 1 y 2 (psicóticos con alucinaciones y psicóticos sin alucinaciones) seguian tratamiento farmacológico con neurolépticos de los grupos butirofenonas y benzamidas fundamentalmente. Uno de los 20 pacientes que formaron estos dos grupos acudia por primera vez a consulta y nunca habia recibido tratamiento psicofarmacológico.

Los pacientes con trastornos de ansiedad recibian tratamiento médico con ansiolíticos del grupo benzodiacepinas. Dos de estos pacientes acudian por primera vez, uno de ellos nunca habia estado a tratamiento psicofarmacológico.

Los pacientes con trastornos afectivos tomaban en el $90 \%$ de los casos antidepresivos tricíclicos, en este grupo se incluye un caso a tratamiento con sales de litio.

La antigüedad de la historia clínica no se tuvo en cuenta dado que sólo uno de los sujetos, diagnosticado de esquizofrenia residual, mostró sintomas evidentes de deterioro.

\section{Instrumentos}

El estado clínico de los pacientes fue valorado por el psiquiatra responsable de los casos (20 años de experiencia) utilizando la Brief Psychiatric Rating Scale (BPRS) de Overall y Gorham (1962). Las razones para la elección de este instrumento pueden sintetizarse en las siguientes:

a) El interés central de la investigación no está tanto en valorar el tipo de alucinaciones que presentan los sujetos como su presencia 0 ausencia.

b) Es un instrumento con garantizada universalidad y estandarización.

c) Es breve y de sencilla aplicación, ideal para contextos ambulatorios donde la mayoría de los pacientes consultan dentro de un promedio que no supera los 10 minutos.

d) Una dispersión de la muestra en función de una sintomatología muy especifica haría imposible la comparación entre grupos dado el elevado número de condiciones experimentales.

Se administró también a cada paciente una prueba de control central creada por los autores, consistente en tres tareas que pretenden valorar la capacidad del paciente para registrar tanto material verbal presentado auditiva y visualmente como, en particular, la fuente (interna o externa) de emisión. Se han utilizado en la segunda tarea de la prueba 20 tarjetas de la Bateria Neuropsicológica de LuriaNebraska (serie M).

\section{Procedimiente}

Los sujetos fueron valorados en consulta ambulatoria, a la que acudian por primera vez o en revisión periódica. La primera de las tareas a realizar por cada paciente consistió en la presentación de una serie de 10 palabras solicitándole al sujeto que a continuación de cada una de ellas generase otra de su invención que comenzase por la última letra de la palabra presentada por el examinador (p. e., mesa-árbol). En la segunda tarea, en este caso sin restricción ortográfica alguna, fueron presentadas sucesivamente las tarjetas de la Bateria Neuropsicológica de Luria-Nebraśka asociando a cada escena representada por la tarjeta una palabra generada por el examinador y por el paciente, alternativamente. En la 
tercera tarea, se presentaron 10 palabras escritas $y$, también de modo alternativo, el paciente debería escribir en otra tarjeta en blanco cualquier palabra que se le ocurriera, pero conceptualmente relacionada con la mostrada por el investigador (p.e., puente-rio). Sin ninguna advertencia previa, las 60 palabras utilizadas en las tres partes de la prueba, 30 generadas por el investigador y 30 generadas por el sujeto, fueron presentadas cinco minutos después de finalizar la prueba (siempre en un orden preestablecido por un sistema de azar) con el fin de que el paciente realizase una tarea de reconocimiento o discriminación entre aquéllas que habian sido generadas por el investigador y las que habian sido generadas por él mismo.

A partir de la elección realizada por cada paciente, fueron obtenidas las siguientes medidas: a) Errores en la atribución interna (EAI): palabras generadas por el sujeto pero que fueron atribuidas al experimentador; b) Errores en la atribución externa (EAE): palabras generadas por el experimentador pero que el sujeto atribuyó a sí mismo; y c) Errores globales (EG): suma de todas las atribuciones erróneas, sin tener en cuenta el sentido de la atribución.

Dichas medidas fueron obtenidas para cada una de las tres tareas, lo que ha permitido obtener $3+(3 \times 3=9)=12$ indicadores. Los sujetos fueron agrupados en las citadas categorías clínicas, en función de la valoración clínica del BPRS y el diagnóstico clínico.

\section{RESULTADOS}

En la Tabla 1 se presentan las correlaciones observadas entre los tres tipos de errores de las tareas realizadas, apreciándose que la correlación más alta se da entre los EAE (errores en la atribución externa) de las subtareas 1 y $3(r=0,54 ; p=0,000)$ siendo la más baja entre los EAl (errores en la atribución interna) de las subtareas 2 y 3 ( $r=0,14$; $p=0,36$ ). Un análisis más detallado de la tabla de correlaciones permite afirmar que los errores en la atribución interna observados en la tarea 2 no son consistentes con los observados en las tareas 1 y 3 . Existe, en cambio, mayor consistencia entre las tres tareas en el tipo de errores de atribución externa y en los errores globales.

La escasa potencia discriminativa de la tarea 2 para determinar las características de los grupos clínicos queda patente también en la comparación de errores observados en cada grupo de pacientes (Tabla 2); a la vez que se comprueba que la tarea 1, en la que se exigía al paciente la generación de palabras sometida a una restricción ortográfica, es la que ha permitido diferenciar significativamente las muestras clínicas.

Dado el escaso tamaño de muestra en relación al número de grupos $(n j=10, p=4)$, se decidió la aplicación de pruebas no paramétricas para detectar las posibles diferencias entre grupos en la tarea de control central.

La comparación de las medias de los diversos tipos de errores cometidos entre los gru-

Tabla 1.- Correlaciones lineales entre los diversos tipos de errores en las tareas 1, 2 y 3

\begin{tabular}{l|lll} 
Tipos de errores & Tareas 1-2 & Tareas 1-3 & Tareas 2-3 \\
\hline $\begin{array}{l}\text { Errores globales } \\
\begin{array}{l}\text { Errores en la } \\
\text { atribución interna }\end{array}\end{array}$ & $\begin{array}{l}0.38 \\
(p<0,01)\end{array}$ & $\begin{array}{l}0.45 \\
(p<0,004)\end{array}$ & $\begin{array}{l}0.35 \\
(p<0,002)\end{array}$ \\
& $\begin{array}{l}0.27 \\
(p<0,09)\end{array}$ & $\begin{array}{l}0.45 \\
(p<0,003)\end{array}$ & $\begin{array}{l}0.14 \\
(p<0,36)\end{array}$ \\
$\begin{array}{l}\text { Errores en la } \\
\text { atribución externa }\end{array}$ & $\begin{array}{l}0.43 \\
(p<0,005)\end{array}$ & $\begin{array}{l}0.54 \\
(p<0,0001)\end{array}$ & $\begin{array}{l}0.34 \\
(p<0,03)\end{array}$
\end{tabular}


Tabla 2.- Comparación de medias en los tipos de errores cometidos por diversos grupos de pacientes.

\begin{tabular}{|c|c|c|c|c|c|}
\hline Grupos & $\begin{array}{l}\text { Variable } \\
\text { dependiente }\end{array}$ & Media & D.T. & $\begin{array}{l}\text { U de Mann- } \\
\text { Whitney }\end{array}$ & $p<$ \\
\hline $\begin{array}{l}1 \text { Psicóticos con alucinaciones } \\
2 \text { Psicóticos sin alucinaciones }\end{array}$ & EAl (tarea 1) & $\begin{array}{l}3,6 \\
1,9\end{array}$ & $\begin{array}{l}1,5 \\
3,0\end{array}$ & 18,5 & 0,01 \\
\hline $\begin{array}{l}1 \text { Psicóticos con alucinaciones } \\
4 \text { Pacientes con trastornos de ansiedad }\end{array}$ & EG & $\begin{array}{r}13,0 \\
9,5\end{array}$ & $\begin{array}{l}5,2 \\
4,11\end{array}$ & 27,0 & 0,08 \\
\hline $\begin{array}{l}1 \text { Psicóticos con alucinaciones } \\
4 \text { Pacientes con trastornos de ansiedad }\end{array}$ & EG (tarea 1) & $\begin{array}{l}5,7 \\
3,7\end{array}$ & $\begin{array}{l}2,5 \\
2,4\end{array}$ & 27,5 & 0,08 \\
\hline $\begin{array}{l}2 \text { Psicóticos sin alucinaciones } \\
4 \text { Pacientes con trastornos de ansiedad }\end{array}$ & EAE & $\begin{array}{l}7,8 \\
3.6\end{array}$ & $\begin{array}{l}5,5 \\
2.5\end{array}$ & 24,0 & 0,04 \\
\hline $\begin{array}{l}2 \text { Psicóticos sin alucinaciones } \\
4 \text { Pacientes con trastornos de ansiedad }\end{array}$ & EAE (tarea 1) & $\begin{array}{l}3.3 \\
1.3\end{array}$ & $\begin{array}{l}2.0 \\
1.1\end{array}$ & 23,5 & 0,03 \\
\hline
\end{tabular}

$E A I=$ Errores en la atribución interna; $E A E=$ Errores en la atribución externa; EG= Errores globales

pos (Tabla 2) permite comprobar que solamente han alcanzado niveles significativos en la dirección predicha en la hipótesis de partida las diferencias en errores en la abribución interna cometidos por los psicóticos con alucinaciones y los psicóticos sin alucinaciones en la tarea 1; en el sentido de que los pacientes que presentaban alucinaciones durante los días anteriores a la valoración cometían más errores en la atribución interna que los pacientes psicóticos sin alucinaciones recientes. Dicho de otra manera, los psicóticos con alucinaciones han tendido con mayor probabilidad a atribuir a si mismos el origen de palabras que habian sido generadas por el experimentador; sin embargo, esta tendencia sólo se ha observado cuando la tarea incluía una limitación ortográfica (lo que, supuestamente, dificultaba la utilización de palabras de contenido abiertamente personalizado o idiosincrático que pudieran facilitar en exceso la discriminación interna-externa).

En segundo lugar, los pacientes psicóticos sin alucinaciones recientes han cometido más errores en la atribución externa (en especial en la tarea 1) que los pacientes con trastornos de ansiedad; es decir, han tendido con más frecuencia a atribuir al experimentador palabras que habian sido generadas por ellos mismos. Este hallazgo, resulta de dificil interpretación, a la luz de las teorias referidas anteriormente, y podria explicarse por posibles problemas atencionales más intensos en los pacientes psicóticos o como consecuencia de otra forma de déficit de discriminación.

Finalmente, se observan más errores globales (sobre todo en la tarea 1) en los pacientes psicóticos con alucinaciones, comparativamente con los pacientes con trastornos de ansiedad, alcanzando niveles próximos a la significación estadistica. Debe tenerse en cuenta, no obstante, que el concepto de error global puede carecer de significado especifico, por tratarse de la suma de los dos tipos de errores antes descritos, y puede deberse también a déficits atencionales más intensos.

En la prueba de Kruskal-Wallis se obtuvo un valor de $c 2[3]=6,06$ con una $p=0,108$ en la variable Eai1 (errores en la atribución interna en la subtarea 1). Con el fin de localizar la comparación/es de medias responsable/s de la significación general, se empleo la t' de Conover (1980) para las 6 comparaciones de medias posibles. Aplicando esta prueba se obtuvo una única significación $\mathrm{t}^{\prime}(\mathrm{N}-\mathrm{p}=36), \mathrm{c}=12,75$ $\left(p=0,05^{\star}\right)$, para la comparación de psicóticos con alucinaciones y psicóticos sin alucinaciones (Tabla 3). Estos datos confirman los resultados de los análisis anteriores utilizando el estadistico $U$ de Mann-Whitney, que apoyan la hipótesis de partida, aunque solamente con la tarea 1. 
Tabla 3.- Diferencias entre rangos en los errores en la atribución interna (subtarea 1), según valores de la $t$ de Conover (1980)

\begin{tabular}{|c|c|c|c|c|c|}
\hline & Rangos & $\begin{array}{l}\text { Psicoticoticos } \\
\text { con alucinaciones } \\
26.4\end{array}$ & $\begin{array}{c}\text { Psicóticos sin } \\
\text { alucinaciones } \\
13,65\end{array}$ & $\begin{array}{c}\text { Pacientes con } \\
\text { trast. afectivos } \\
21,55\end{array}$ & $\begin{array}{l}\text { Pacientes con trast. } \\
\text { de ansiedad } \\
20,4\end{array}$ \\
\hline $\begin{array}{l}\text { Psicoticos con } \\
\text { alucina.ciones }\end{array}$ & 26,4 & - & $12,75^{*}$ & 4,85 & 6.4 \\
\hline $\begin{array}{l}\text { Psicoticos sin } \\
\text { alucinaciones }\end{array}$ & 13,65 & - & - & 7,9 & 6,75 \\
\hline $\begin{array}{l}\text { Pacientes con } \\
\text { trast. afectivos }\end{array}$ & 21,55 & - & - & - & 1,15 \\
\hline $\begin{array}{l}\text { Pacientes con } \\
\text { trast.de ansiedad }\end{array}$ & 20,4 & - & - & - & - \\
\hline
\end{tabular}

" $p=0,05$ para $t$ ' con $[(N-p), c]$ g.l.

\section{DISCUSIÓN}

De las tres partes del estudio realizado con la prueba de control central, puede concluirse que solamente la primera de ellas, que exige la producción verbal por el paciente con el condicionante de la restricción ortográfica permite detectar diferencias entre los pacientes psicóticos con alucinaciones auditivas recientes y sin alucinaciones. El sentido de la comparación indica que los pacientes que experimentan alucinaciones atribuyen con mayor probabilidad las palabras autogeneradas a una fuente externa (en este caso, al experimentador); lo que está en consonancia con la hipótesis de Frith $(1987,1992)$, que atribuye dichos fenómenos alucinatorios a un déficit en la control central o en la autoconsciencia sobre los propios pensamientos, intenciones, deseos, etc. Esta tendencia se ha observado en las tres tareas realizadas, si bien sólo en la primera alcanza significación estadistica.

Resultados coincidentes son los presentados por Harvey y Serper (1990) o Bentall, Baker y Havers (1991).

La naturaleza de la segunda y tercera tareas no ha permitido ratificar los resultados anteriores con la suficiente confianza estadistica, aun tratándose de actividades muy similares a la primera. Podría pensarse, a modo de explicación, que la asociación de las palabras a una imagen fotográfica (tarea 2) o la presentación escrita de las palabras $y$, a su vez, la producción escrita de palabras por el paciente (tarea 3) constituyen variaciones que podrían facilitar la autoconsciencia o el juicio respecto a su fuente de emisión, al contener peculiaridades idiosincráticas, así como un feedback visual y motor asociado a la producción verbal, que darían lugar a una codificación diferenciada de las palabras propias y ajenas; lo que favorecería su posterior reconocimiento inmediato. Por el contrario, la tarea 1 posiblemente dificulta la utilización de estrategias personales de codificación; lo que pondria en mayor medida en evidencia el supuesto déficit del control central en el posterior reconocimiento.

Una segunda conclusión es que los pacientes psicóticos sin alucinaciones recientes cometen más errores en la atribución externa, comparativamente con los pacientes con trastornos de ansiedad. Los pacientes con trastornos afectivos ocupan una posición intermedia en el número de errores en la atribución externa, aunque sin diferencias estadisticamente significativas con los otros dos grupos. Se 
ha invocado más arriba la posibilidad de que dichos errores puedan deberse meramente a deficiencias atencionales inespecificas, en la medida en que estos déficits generalmente se han encontrado en este mismo orden de gravedad en estos subgrupos de pacientes; siendo más intensos en los psicóticos, seguidos de los pacientes con trastornos afectivos y de los pacientes problemas de ansiedad (Boker, Brenner \& Wurgler, 1989; Gibbons, 1990; Nuechterlein \& Dawson, 1984; Rosenbaum, Shore \& Chapin, 1988).

Al no existir diferencias significativas generalizadas del grupo de sujetos con alucinaciones con respecto al grupo de trastornos afectivos y trastornos de ansiedad indica la baja especificidad de la tarea. Resultados similares para otros indicadores cognitivos han sido encontrados por Hollander, Stein, De Caria et al. (1992).

Por otro lado, Johnson y Raye (1981) argumentan que las tareas de control sin restricción, son más fáciles que aquellas que llevan limitación ortográfica (estudios realizados sobre población normal). A tenor de lo dicho anteriormente, si el grupo psicóticos con alucinaciones auditivas presenta especiales dificultades en esta subtarea debemos deducir que esto se debe a las caracteristicas de la misma. Sin embargo, ¿podemos suponer que este tipo de tarea resta control voluntario al sujeto siendo ahí precisamente donde muestran dificultades los psicóticos con alucinaciones?, ¿estamos eliminando los trazos de que disponen este tipo de sujetos para guiar la información en su particular estilo de procesamiento?.

En conclusión, se hace necesario establecer tareas sobre un marco experimental que permitan el estudio de las características semánticas del discurso interno en pacientes psicóticos con alucinaciones auditivas. De cualquier modo estas son cuestiones que quedan planteadas de cara a nuevos trabajos sobre nuevos datos.

Partiendo de la suposición de que las alucinaciones verbales son un fenómeno continuo, que va desde los automensajes del pensamiento hasta la sonorización completa de los mismos, es decir, hasta la verbalización como tal, es posible que las alucinaciones verbales sean pensamientos ruidosos $y$, por tanto, consistan en un aumento gradual de los propios automensajes del sujeto. En este sentido, Collicut y Hemsley (1981) aplican la ley de Weber al fenómeno de las alucinaciones. La ley de Weber enuncia que: la diferencia de sonoridad que se requiere para que ésta resulte perceptible (umbral diferencial) "Dx" es proporcional a la intensidad " $x$ " que en ese instante posea el estímulo " $D x=k x^{\prime \prime}$.

Resultaria relevante conocer el umbral diferencial de sonoridad, si y sólo si, la diferencia de sonoridad fuere determinante en la dilucidación externo-interno. Si esto ocurriera asi, podriamos hablar de umbral diferencial de externalidad/internalidad. Esta objeción, es sustancialmente distinta a la presentada por Slade y Bentall (1988) que achacaron los resultados negativos de esta y otras investigaciones a que el proceso de control es esencialmente cualitativo, reduciéndolo al estatus de juicio categórico: externo/interno. Tal asunción, básicamente correcta, no es incompatible con la idea de que aunque el control central sea un acto judicativo, deba dar cuenta de un fenomeno gradual y por ende, pueda producirse gradualmente. Si esto se demuestra que es asi, es decir, que la decisión sobre el origen de la fuente estimular que recibe el organismo depende del grado de sonorización del pensamiento $y$, por tanto, este proceso se produce gradualmente, el acto judicativo externol interno habria sido dicotomizado artificialmente. Desgraciadamente, las cosas no son $\tan$ simples. Si lo apostillado en estas últimas lineas fuera cierto, sucederia que cualquier sujeto normal alucinaria un gran número de veces al dia, coincidiendo con aquellas en las que habla en voz alta. Dado que, naturalmente, esto no es así, concluiremos que el grado de sonorización es indicativo de externalidad/ internalidad, al menos en parte, ya que únicamente informa al sujeto del nivel de articulación de su discurso; en términos de juicio, si está pensando o hablando (llevado el proceso a sus últimas consecuencias).

Podria pensarse que toda esta enjundia metateórica no conduce a buen puerto y resulta tan engorrosa como la que otrora hiciera Freud. Nada más lejos de lo real. A partir de lo antes expuesto, podríamos interrogarnos: ¿que ocurriria si el organismo, además de ser informado acerca del nivel de articulación del discurso (distinción pensado/hablado), pose- 
yera información acerca de la voluntariedad de tal acción?. La respuesta merece ser objeto de análisis; si bien se apuntan a continuación los pasos que pudieran componer la función de control central: a) secuenciación: planificación, organización del discurso y pautación motora que ha originado la elicitación sensorial (esto último puede proporcionar las claves para la memoria y consciencia de control); b) sonorización: establecimiento del nivel de articulación del discurso en base al grado de simbolización/fonetización; y c) corolarización: información acerca de la voluntariedad/involuntariedad de la acción, dada su presencia/ausencia.

Estudios recientes sobre esquizofrenia, se centran en la detección de indicadores neuropsicológicos en población infantil, donde parece ser que el comportamiento discriminativo de estos marcadores es más potente (Asarnow, Asamen, Granholm, Sherman, Watkins y Williams, 1994). Lo realmente esperanzador de estas investigaciones radica en la necesidad de valorar justamente los factores madurativos. Piaget (1974), proporcionó una explicación evolutiva y conductual de la representación mental que bien podría aplicarse a investigaciones futuras en el sentido arriba presentado. El origen de la simbolización, según Piaget, tiene lugar en conductas motoras externas que el niño internaliza progresivamente entre los 18 y 24 meses de vida.

El papel motor en la formación de imágenes, su protagonismo en los trazos de memoria, su participación decisiva en el feedback que nos conecta con el control (voluntario/ involuntario) de la acción y, por tanto, su estrecha filiación con la consciencia debe estar presente en un marco teórico fuerte. Todo ello puede o no tener que ver con los potentes fármacos de acción bloqueadora sobre los receptores dopaminérgicos y sus efectos extrapiramidales. En todo caso, estas relaciones son aún opacas y deberán ser explicadas si se quiere hablar de causas en cuanto al origen de las experiencias psicóticas.

\section{REFERENCIAS BIBLIOGRÁFICAS}

Asarnow, R. F., Asamen, J., Granholm, E., Sherman, T., Watkins, J. M., \& Williams, M. E. (1994). Cognitive/neuropsychological studies of children with a schizophrenic disorder. Schizophrenia bulletin, 20, 647-670.

American Psychiatric Association (1994). Diagnostic and statistical manual of mental disorders. Washington, DC: APA.

Bentall, R. P., Baker, G. A., \& Havers, S. (1991). Reality monitoring and psychotic hallucinations. British Journal of Clinical Psychology, 30, 213222.

Boker, W., Brenner, H. D., \& Wurgler, S. (1989). Vulnerability-linked deficiencies, psychology and coping behaviour of schizophrenics and their relatives. British Journal of Psychiatry, 155 (Suppl 5), 128-135.

Collicut, J. R., \& Hemsley, D. R. (1981). A psychophysical investigation of auditory functioning in schizophrenia. British Joumal of Clinical Psychology, 20, 199-204.

Conover, W.V. (1980). Practical Nonparametric Statistics. (2nd Edition). New York: John Wiley.

Freud, S. (1895). Proyecto de una psicología para neurólogos y otros escritos. Madrid: Alianza Editorial (Orig. 1895).

Frith, C. D. (1987). The positive and negative symptoms of schizophrenia reflect impairments in the perception and initiation of action. Psychological Medicine, 17, 631-648.

Frith, C. D. (1992). The cognitive neuropsychology of schizophrenia. Hove, UK: Erlbaum.

Frith, C. D., \& Done, D. J. (1988). Towards a neuropsychology of schizophrenia. British Journal of Psychiatry, 153, 437-443.

Frith, C. D., \& Done, D. J. (1989). Experiences of alien control in schizophrenia reflect a disorder in the central monitoring of action. Psychological Medicine, 19, 359-363.

Gibbons, F. X. (1990). Self-evaluation and selfperception: The role of attention in the experience of anxiety. Anxiety Research, 2, 153163.

Gray, J. A., Feldon, J., Rawlins, J. N. P., Hemsley, D. R., \& Smith, A. D. (1991). The neuropsychology of schizophrenia. Behavioral and Brain Sciences, 14, 1-84.

Harvey, P. D. (1985). Reality monitoring in mania and schizophrenia. Nervous and Mental Disease, 173 (2), 67-73.

Harvey, P. D., \& Serper, M. R. (1990). Linguistic and cognitive failures in schizophrenia: $A$ multivariate analysis. Journal of Nervous and Mental Disease, 178 (8), 487-493.

Hemsley, D. R. (1993). A simple (or simplistic?) cognitive model for schizophrenia. Behavioural Research Therapy, 31, 663-645.

Hoffman, R. E. (1986). Verbal hallucinations and language production processes in schizophrenia. The Behavioral and Brain Sciences, 9, 503-548. 
Hollander, E., Stein, D. J., DeCaria, C. M., Klein, D. F., \& Liebowitz, M. R. (1992). Neuropsychiatry and 5-HT function in OCD and social phobia.

James, W. (1989). Principios de Psicologia. México, D.F.: Fondo de Cultura Económica (Orig. 1890).

Johnson, M. K., \& Raye, C. L. (1979). Fact and fantasy: The roles of accuracy and variability in confusing imaginations with perceptual experiences. Joumal of Experimental Psychology: Human Learning and Memory, 5, 229-240.

Johnson, M. K., \& Raye, C. L. (1981). Reality monitoring. Psychological Review, 88, 67-85.

Johnson, M. K., Hashtroudi, S., \& Lindsay, D. S. (1993). Source monitoring. Psychological Bulletin, 114, 3-28.

Johnson, M. K., Raye, C. L., \& Durso, F. T. (1980). Reality monitoring: second perceptions and thoughts. Bulletin of the Psychonomic Society, 15, 402-404.

Johnson, M. K., Taylor, T. H., \& Raye, C. L. (1977). Fact and fantasy: the effects of internally generated events on the apparent frequency of externally generated events. Memory and Cognition, 5, 116-122.

Johnson, M. K., Raye, C. L., Hasher, L., \& Chromiak, W. (1979). Are there developmental differences in reality monitoring? Journal of Experimental Child Psychology, 27, 120-128.

Mlakar, J., Jensthrle, J., \& Frith, C. D. (1994). Central monitoring deficiency and Schizophrenic Symptoms. Psychological Medicine, 24, 557 564.

Nuechterlein, K. H., \& Dawson, M. E. (1984). Information processing and attentional functio- ning in the developmental course of schizophrenic disorders. Schizophrenia Bulletin, 10, 160-203.

Organización Mundial de la Salud (1992). Trastornos mentales y del comportamiento: descripciones clínicas y pautas para el diagnóstico. (10 ed.). Madrid: Meditor.

Overall, J.E. y Gorham, D.R. (1962) The Brief Psychiatric Rating Scale. Psychological Reports, 10, 799-812.

Peters, E. R., Pickering, A. D., \& Hemsley, D. R. (1994). Cognitive inhibition and positive symptomatology in schizotipy. British Joumal of Clinical Psychology, 33, 33-48.

Piaget, J. (1974). The child and reality. London: Muller.

Raye, C. L., \& Johnson, M. K. (1980). Reality monitoring vs. discriminating between external sources of memories. Bulletin of the Psychonomic Society, 15, 405-408.

Raye, C. L., Johnson, M. K., \& Taylor, T. H. (1980). Is there something special about memory for internally generated information? Memory and Cognition, 8, 141-148.

Rosenbaum, G., Shore, D. L., \& Chapin, K. (1988). Attention deficit in schizophrenia and schizotypy: Marker versus symptom variables. Joumal of Abnormal Psychology, 97, 41-47.

Slade, P. D. (1976). Towards a theory of auditory hallucinations: outline of an hypothetical fourfactor model. British Joumal of Social and Clinical Psychology, 13, 73-79.

Slade, P. D., \& Bentall, R. P. (1988). Sensory deception. London: Croom Helm.

Tuke, D. H. (1889). Hallucinations, and the subjetive sensations of the sane. Brain, 11, 441-467.

\section{NOTA}

Agradecimientos. La realizacion del presente estudio ha sido posible gracias a la ayuda TA94/206-2 concedida a los autores por la Universidad de Oviedo. Los autores desean expresar su agradecimiento a los Dres. C.D. Frith por sus valiosos consejos y G. Vallejo por su apoyo metodologico. 